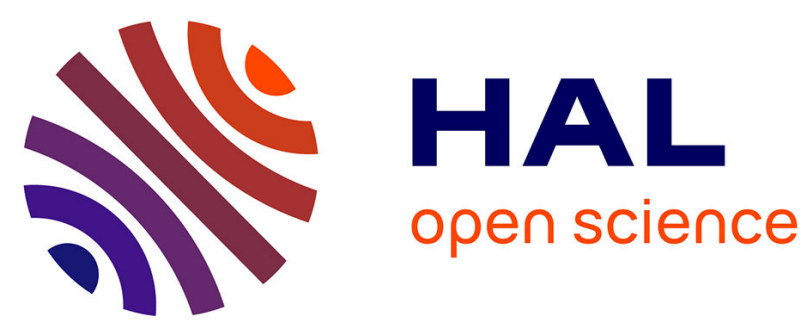

\title{
From Enynyl Esters to Functionalized Polycyclic Derivatives via Cycloisomerization and Post-Functionalization
}

Simon Guelen, Max Blazejak, Virginie Mouriès-Mansuy, Louis Fensterbank

\section{- To cite this version:}

Simon Guelen, Max Blazejak, Virginie Mouriès-Mansuy, Louis Fensterbank. From Enynyl Esters to Functionalized Polycyclic Derivatives via Cycloisomerization and Post-Functionalization. Synthesis: Journal of Synthetic Organic Chemistry, 2016, 48 (19), pp.3199 - 3206. 10.1055/s-0035-1561488 . hal-01400636

\section{HAL Id: hal-01400636 \\ https://hal.sorbonne-universite.fr/hal-01400636}

Submitted on 22 Nov 2016

HAL is a multi-disciplinary open access archive for the deposit and dissemination of scientific research documents, whether they are published or not. The documents may come from teaching and research institutions in France or abroad, or from public or private research centers.
L'archive ouverte pluridisciplinaire HAL, est destinée au dépôt et à la diffusion de documents scientifiques de niveau recherche, publiés ou non, émanant des établissements d'enseignement et de recherche français ou étrangers, des laboratoires publics ou privés. 


\section{From Enynyl Esters to Functionalized Polycyclic Derivatives via Cycloisomerization and Post-Functionalization}

\author{
Simon Guelen \\ Max Blazejak \\ Virginie Mouriès-Mansuy* \\ Louis Fensterbank* \\ Institut Parisien de Chimie Moléculaire, UMR CNRS 8232, \\ Sorbonne Universités UPMC Univ Paris 06. 4 Place Jussieu, \\ CC 229, F-75252 Paris Cedex 05, France \\ virginie.mansuy@upmc.fr; louis.fensterbank@upmc.fr \\ Dedicated to Jean F. Normant with admiration
}

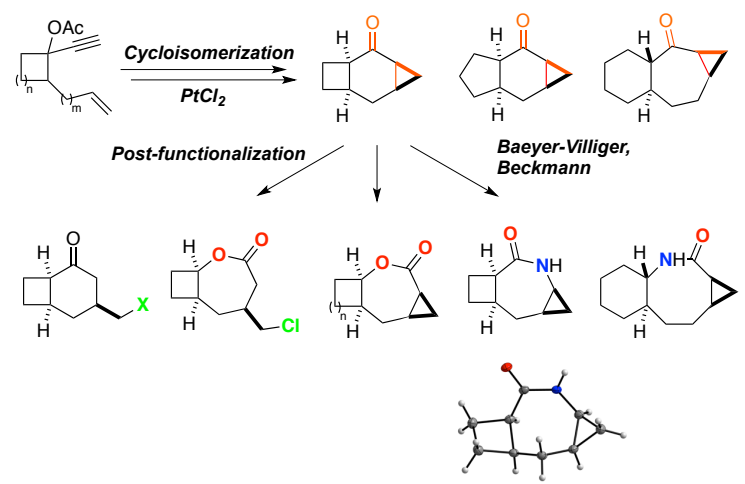

\begin{abstract}
Received: Published online: Dol

Abstract: The post-functionalization of ketone products originating from the $\mathrm{PtCl}_{2}$-catalyzed cycloisomerization of enynyl esters is described. Postfunctionalization has been accomplished via diastereoselective alkylation, regioselective cyclopropane opening and regioselective Baeyer Villiger and Beckmann rearrangements to provide functionalized polycycles.
\end{abstract}

Key words: cycloisomerization, platinum, enynyl ester, polycyclic ketone, Baeyer-Villiger, Beckmann rearrangement, lactones, lactams

Since the serendipitous rediscovery by us in $2002^{1}$ of the $\mathrm{PtCl}_{2}$ catalyzed 1,2-OAc migration of propargyl acetates to generate platinum carbenoid intermediates, ${ }^{2}$ this chemistry has witnessed intense investigations notably for conducting valuable enyne cycloisomerizations leading to molecular complexity ${ }^{3}$ from readily available precursors. ${ }^{4}$ The possibility to use gold ${ }^{5}$ and more rarely other metals such as rhodium as catalysts, ${ }^{6}$ in each case bringing in their specific features, have greatly contributed to these developments.

The tandem 1,2-OAc migration-intramolecular cyclopropanation of substrates $\mathbf{1}$ to polycycles $\mathbf{2}$ is one of the iconic synthetic sequences that has been extensively studied by several groups ${ }^{7}$ including ours. ${ }^{1,8}$ Besides intriguing mechanistic manifolds, ${ }^{9}$ the carbocyclic platforms obtained in a highly diastereoselective manner are rich in synthetic potential, notably after a hydrolysis treatment which liberates versatile ketone derivatives $\mathbf{3}$ (Scheme 1). Herein, we focus on a series of these ketone substrates and notably examine their post functionalization leading to various types of functionalized products.

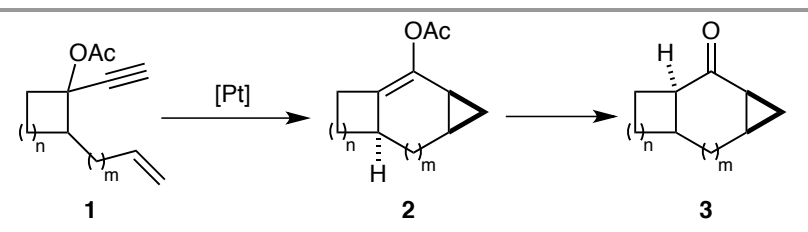

Scheme 1 Cycloisomerization of monocyclic enynyl esters

Synthesis of the platforms was carried out following the literature route (Scheme 2). ${ }^{10}$ Starting from the corresponding cyclic ketones A, allyl cyclobutyl (1a), allyl cyclopentyl (1b) enyne acetate as well as the cyclohexyl derivative bearing a homoallyl group (1c) were obtained in a five step synthesis (formation of dimethylhydrazone, alkylation and hydrolysis, addition of TMS-acetylene, cleavage of TMS group and acetylation). All three were then cyclized to the tricyclic enol acetates 2a-c using $5 \mathrm{~mol} \% \mathrm{PtCl}_{2}$ and saponified with potassium carbonate in methanol to the ketones 3a-c in excellent yields and as single diastereomers. ${ }^{8 \mathrm{~d}}$

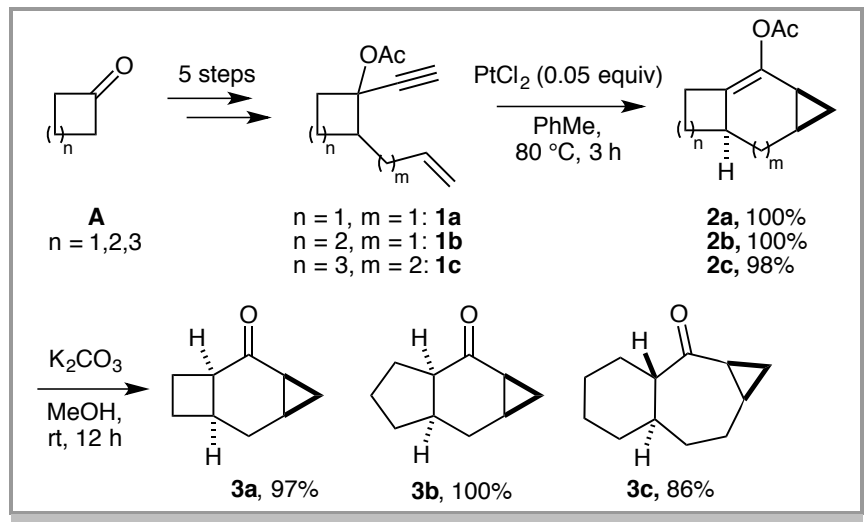

Scheme 2 Synthesis of ketones $3 a-c$ 
In order to establish the relative stereochemistry of these derivatives, ketones 3a and 3c, which were obtained as oils, were then condensed with 2,4-dinitrophenylhydrazine in refluxing methanol in the presence of $p$-toluenesulfonic acid to give the corresponding 2,4-dinitrophenylhydrazones 3a-DNPH and 3c-DNPH as crystalline solids in $68 \%$ and $71 \%$ yield respectively. Structure and relative configuration of these compounds, and thus of $\mathbf{3 a}$ and $\mathbf{3 c}$, were confirmed through single crystal X-ray analysis (Figure 1). ${ }^{11}$

It is noticeable, that in 3a all four bridging hydrogens are cisconfigured with a syn-configuration of the four- and threemembered ring, whereas in 3c the bridging hydrogens of the six-membered ring are in trans-configuration. This suggests that during the enol acetate hydrolysis the ring strain confines the four-membered ring in $\mathbf{3 a}$ to give the cis-isomer, while the sixmembered ring of $\mathbf{3 c}$ is large and flexible enough to form the more stable trans-isomer with a perfect chair conformation. Based on the fact that the favored ring junction between a cyclohexanone and a five-membered ring is $c i s,{ }^{12}$ we hypothesized the same for $\mathbf{3 b}$ and thus a relative stereochemistry as seen for $\mathbf{3 a}$.

Because of its very specific structure featuring marked concave and convex faces, we looked at addition reactions on the carbonyl moiety anticipating highly diastereoselective processes. To probe this, we looked at the 1,2-addition of a lithium acetylide. After deprotonation with $n$-BuLi, phenylacetylene reacted with $\mathbf{3 a}$ to give alcohol $\mathbf{4}$ in $61 \%$ yield, as a single diastereomer (Scheme 3). Its relative stereochemistry was established by NOESY of the corresponding $O$-allyl (4-0allyl) derivative and confirmed an exclusive attack from the convex face.

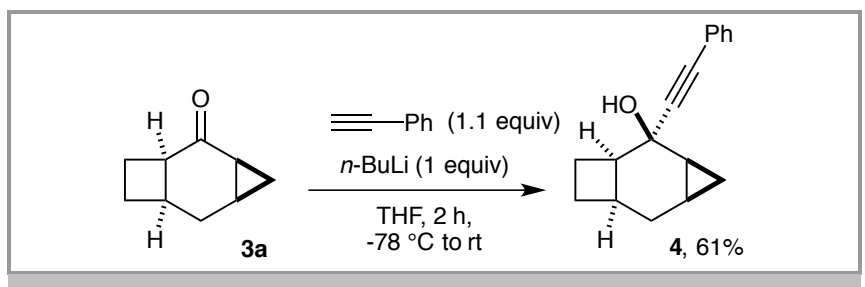

Scheme 3 Diastereoselective alkylation of ketone 3 a

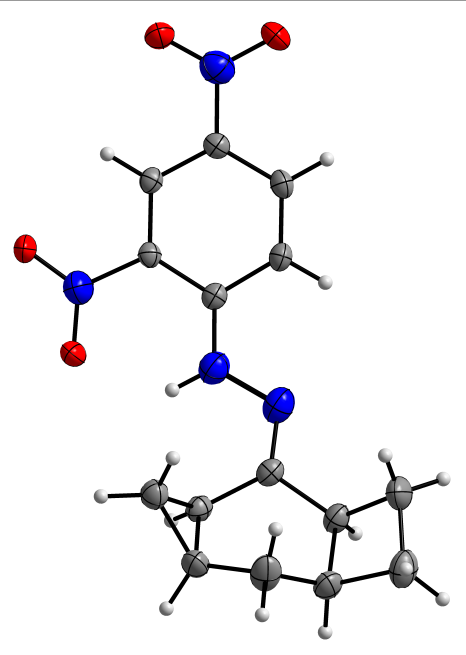

a)

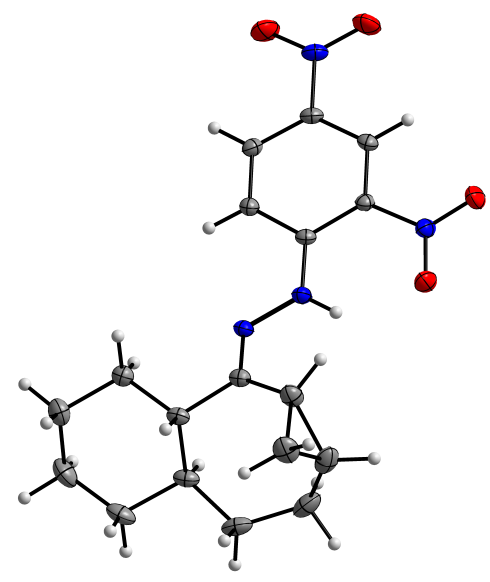

b)

Figure 1 a) Crystal structure of $3 a-D N P H$, showing the cis-configuration of all four bridging hydrogen atoms. b) Crystal structure of 3c-DNPH

After derivatizing the carbonyl moiety, we focused our attention on the cyclopropyl moiety. Indeed, a potentially valuable way to introduce functionality onto the platform would be the selective opening of the strained cyclopropyl moiety with halides. Interestingly, Tardella and coworkers used pyridinium hydrochloride as halide source to open a related bicyclic ketone in a selective manner. ${ }^{13}$ In our hands, using pyridinium salts as halide source (Scheme 4), a C-C cleavage reaction took place cleanly to give the respective bicyclic chloro- and bromoketones $\mathbf{5}$ and $\mathbf{6}$ as single diastereomers. 

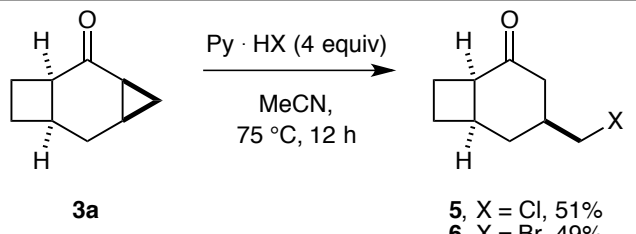

5, $\mathrm{X}=\mathrm{Cl}, 51 \%$ 6, $\mathrm{X}=\mathrm{Br}, 49 \%$
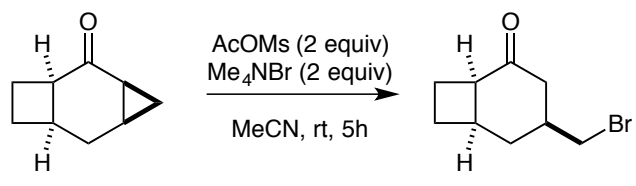

$3 a$

$6,54 \%$

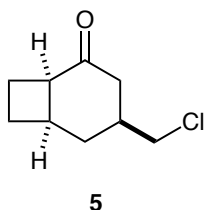

$\underset{\mathrm{CH}_{2} \mathrm{Cl}_{2}, \mathrm{rt}, 12 \mathrm{~h}}{\stackrel{m \mathrm{CPBA}}{\longrightarrow} \text { (2.4 equiv) }}$

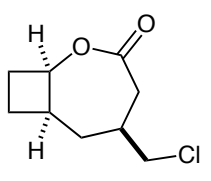

7, $84 \%$

Scheme 4 Regioselective cyclopropyl opening of ketone $3 a$ and subsequent Baeyer-Villiger oxidation

Although the yields were only average, other tested methods did not prove to be more successful. Acetylmethanesulfonate, which in combination with tetramethylammonium bromide had also been used successfully in the literature ${ }^{14}$ as an activating agent to open cyclopropyl ketones for example, gave bromide 6 in $54 \%$ yield. Further heterofunctionalization could be brought on chloroketone $\mathbf{5}$ through a regioselective Baeyer-Villiger (BV) reaction to give lactone $\mathbf{7}$ in good yield.

From a regioselectivity point of view, the BV oxidation of 3a appeared highly intriguing. It had been shown by the group of Wiberg, ${ }^{15}$ that this reaction is completely regioselective for the bicyclic ketone cyclopropyl-cyclopentanone, where only the cyclopropyl group is migrating (Scheme 5).

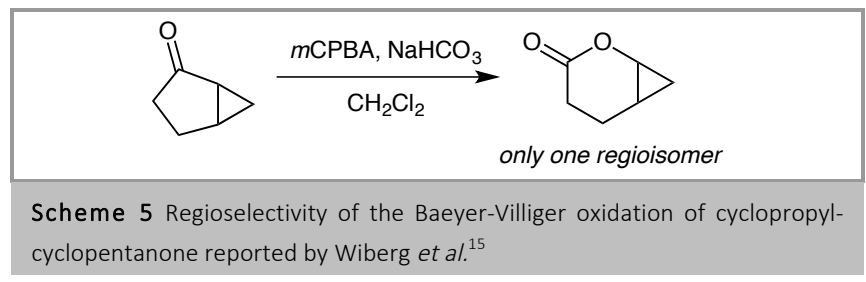

$\mathrm{BV}$ reactions of $\mathbf{3 a}$ and $\mathbf{3} \mathbf{b}$ were thus carried out in standard conditions using $m C P B A$ in dichloromethane (Scheme 6). Gratifyingly, lactones $\mathbf{8}$ and $\mathbf{9}$ were formed as single regioisomers, which structures could be assigned by 2D NMR. Consistent with Wiberg's results, only the larger cyclobutyl (8a) and cyclopentyl (8b) groups migrated.

Surprisingly, 3c did not react at all under identical BV oxidation conditions and only starting material was recovered. ${ }^{16}$ Although the reason for this remains unclear, it might be possible that the formation of an eight-membered ring lactone is unfavorable. The different configuration of the ring junction of $\mathbf{3 c}$ compared to $\mathbf{3 a}$ and $\mathbf{3} \mathbf{b}$ could also play a role.

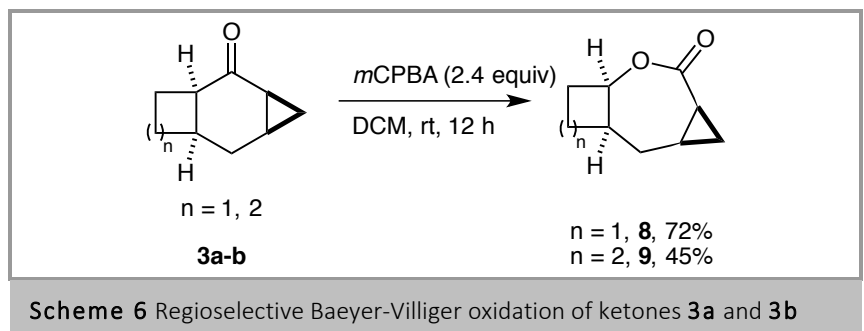

To compare the BV oxidation to another ring expansion reaction, we explored the possibility of a Beckmann rearrangement, which would convert, after oxime formation, the cyclic ketone to the corresponding lactames. ${ }^{17}$ At first, this task seemed challenging, as in several experiments using hydroxylammonium chloride and sodium acetate in methanol no oxime at all was formed. Here a switch to different bases and solvent systems helped. Oxime $\mathbf{1 0}$ could be synthesized using pyridine in ethanol, $\mathrm{NaOH}$ in ethanol-water or with sodium carbonate in ethanol-water, which gave the best yield (51\% of 10, see Scheme 7).

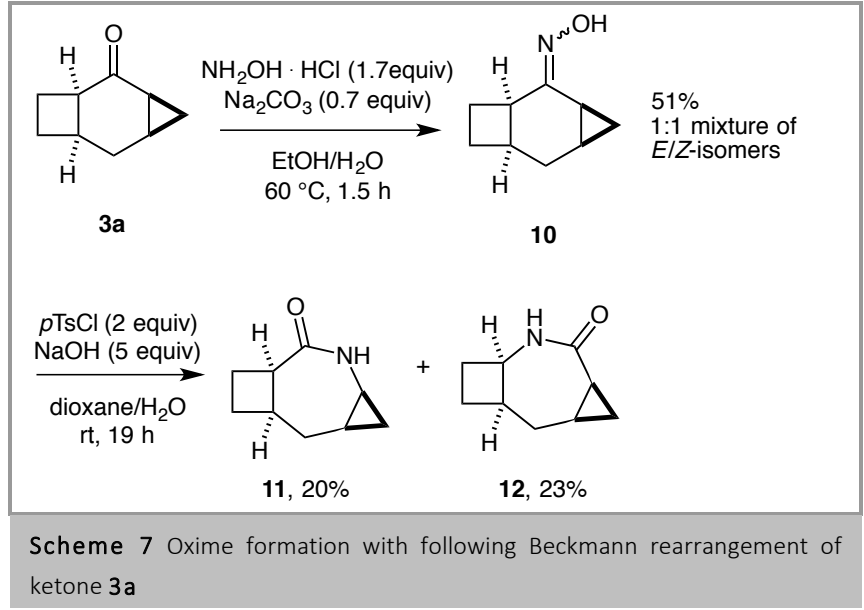

The oxime $\mathbf{1 0}$ was obtained as an inseparable 1:1 mixture of E/Z-isomers. Beckmann rearrangement then proceeded smoothly.18 Although the rearrangement itself is probably regiospecific, starting from $\mathbf{1 0}$ two regioisomeric amide products $\mathbf{1 1}$ and $\mathbf{1 2}$ were obtained. As they showed a surprisingly large difference in polarity, they were easily separable by chromatography. Amide $\mathbf{1 1}$ gave crystals suitable for X-ray diffraction analysis, ${ }^{19}$ which allowed for an unequivocal assignment of both isomers (Figure 2). A direct Beckmann rearrangement starting from 3a with $\mathrm{NH}_{2} \mathrm{SO}_{3} \mathrm{H}$ in formic acid was possible, too, but gave slightly lower yields. ${ }^{20}$ 


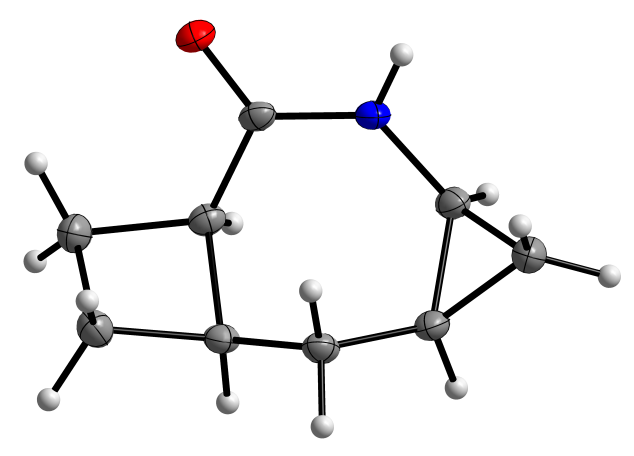

Figure 2 Crystal structure of 11

With 3c, which as mentioned before did not react under the BV conditions, oxime formation and Beckmann rearrangement were possible (Scheme 8).

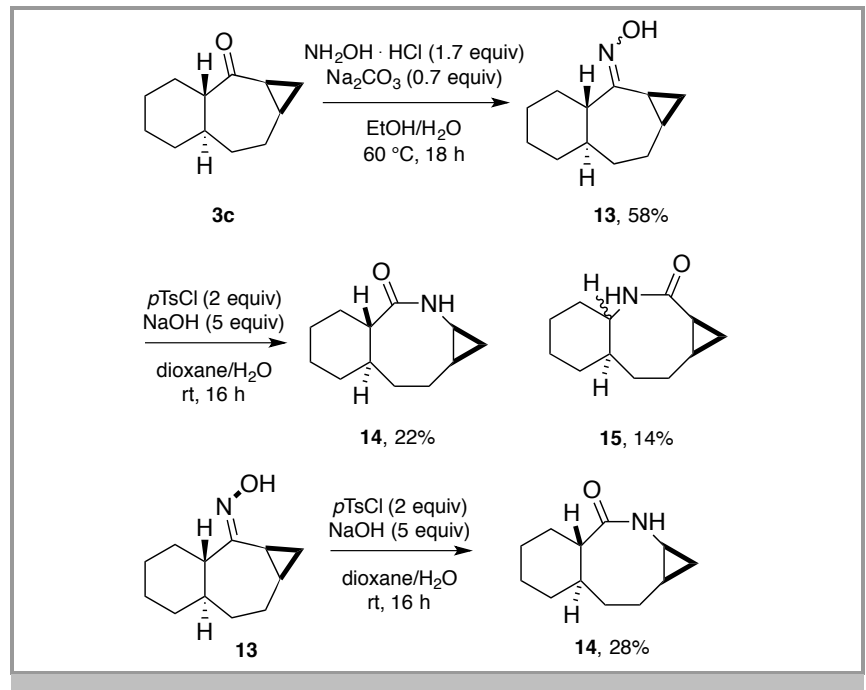

Scheme 8 Oxime formation and Beckmann rearrangement of ketone $3 \mathrm{c}$

Again, oxime 13 was obtained as a 1:1 mixture of both $(E)$ - and $(Z)$-isomer, and although they were not completely separable, this time some of the more polar oxime could be isolated by column chromatography. While the (E/Z)-mixture of 13 , as before, yielded two regioisomeric amides 14 and 15 (as a mixture of diastereomers) in the Beckmann rearrangement, oxime 13 as a single diastereomer gave only 14 in 28\% yield. This strongly hints that the reaction is regiospecific with respect to the oxime conformation.

In summary, based on our works on catalytic enyne cycloisomerizations, structurally interesting tricyclic ketones were synthesized, fully characterized and studied as a precursor for regio- and diastereoselective reactions. Nucleophilic attack of the tricyclic ketone by a lithium acetylide turned out to be completely diastereoselective. Selective cyclopropyl opening in the 3-position was easily possible using a pyridinium salt as halide source. The well-known Baeyer-Villiger ring expansion reaction showed full regioselectivity and only migration of the larger ring occured. Beckmann rearrangement from the corresponding oximes could provide valuable polycyclic amides.

\section{The experimental section has no title; please leave this line here.}

Unless otherwise noted, reactions were carried out under argon atmosphere. Methanol was dried overnight over freshly activated molecular sieves (4 Å), THF and diethyl ether were distillated from sodium/benzophenone. Other reagents and chemicals were purchased from commercial sources and used as received. Infrared (IR) spectra were recorded on a Bruker Tensor 27 (ATR diamond) spectrophotometer. Melting points were determined on a melting point apparatus SMP3 (Stuart scientific) and are uncorrected. NMR spectra were recorded at room temperature on Bruker AVANCE 600, 400 or 300 spectrometers. Chemical shifts $(\delta)$ are reported in parts per million (ppm) and coupling constants $\left(U\right.$ ) are given in Hertz (Hz). ${ }^{1} \mathrm{H}$ and ${ }^{13} \mathrm{C}$ NMR assignments were based on COSY, HSQC and HMBC experiments. Abbreviations used for peak multiplicity are: s (singlet); bs (broad singlet); d (doublet); t (triplet); q (quartet); p (quintuplet), m (multiplet) and for assignments: cp (cyclopropyl); cb (cyclobutyl); cpe (cyclopentyl); ch (cyclohexyl). High resolution mass spectrometry was performed on a microTOF (ESI). Thin layer chromatography (TLC) was performed on Merck silica gel $60 \mathrm{~F} 254$ and detected with a UV lamp $(\lambda=254 \mathrm{~nm})$ and $\mathrm{KMnO}_{4}$ or $p$-anisaldehyde staining. Flash column chromatography was performed on silica Geduran® Si $60 \AA ̊(40-63 \mu \mathrm{m})$.

\section{Compounds 3a-c; General Procedure}

To a stirred solution of the corresponding tricyclic acetate ${ }^{8 \mathrm{~d}} \mathbf{2 a - c}$ (1 equiv.) in dry methanol ( $0.15 \mathrm{M})$ at rt under argon was added potassium carbonate ( 2 equiv.) portion wise and the mixture was stirred at rt for 12 h. The reaction mixture was quenched with saturated ammonium chloride solution. The aqueous phase was extracted three times with diethylether. The combined organic layers were washed with brine, dried over $\mathrm{MgSO}_{4}$ and concentrated under reduced pressure to give the desired product.

\section{( \pm )-(3R,5R)-tricyclo[5.2.0.0 3,5$]$ nonan-2-one (3a)}

Scale: 3.8 g, 21.3 mmol; Yield: 2.83 g (97\%); Brown oil; $R_{f}=0.11$ (petroleum ether/EtOAc, 95:5).

IR (ATR): 2924, 2847, 1683, 1663, 1440, 1349, 1251, 1009, 934, 839, $745 \mathrm{~cm}^{-1}$.

${ }^{1} \mathrm{H} \mathrm{NMR}\left(400 \mathrm{MHz}, \mathrm{CDCl}_{3}\right) \delta=2.97-2.92(\mathrm{~m}, 2 \mathrm{H}), 2.49-2.42(\mathrm{~m}, 1 \mathrm{H}), 2.32-$ $2.25(\mathrm{~m}, 1 \mathrm{H}), 1.98-1.90(\mathrm{~m}, 3 \mathrm{H}), 1.85-1.79(\mathrm{~m}, 2 \mathrm{H}), 1.73-1.71(\mathrm{~m}, 1 \mathrm{H})$, 1.25-1.21 (m, 1H), 0.91-0.84 (m, 1H).

${ }^{13} \mathrm{C}$ NMR (100MHz, $\mathrm{CDCl}_{3}$ ) $\delta=213.1,41.4$ (CCO cb), 32.3, 25.1, 25.0, 23.5, 22.4, 21.9 (CCO cp), 13.9.

HRMS (ESI): $\mathrm{m} / \mathrm{z}[\mathrm{M}+\mathrm{Na}]^{+}$calcd for $\mathrm{C}_{9} \mathrm{H}_{12} \mathrm{ONa}$ : 159.0780; found: 159.0779 .

\section{( \pm )-(1aR,6aR)-octahydrocyclopropa[f]inden-2(1H)-one (3b)}

Scale: $0.15 \mathrm{~g}, 0.78 \mathrm{mmol}$; Yield: $0.12 \mathrm{~g}$ (quant); Yellow oil; $\mathrm{R}_{\mathrm{f}}=0.4$ (petroleum ether/EtOAc, 90:10).

IR (ATR): 2959, 2856, 1689, 1366, 1234, $885 \mathrm{~cm}^{-1}$.

${ }^{1} \mathrm{H}$ NMR $\left(400 \mathrm{MHz}, \mathrm{CDCl}_{3}\right) \delta=2.36$ (ddd, $\left.J=13.4,7.8,5.2 \mathrm{~Hz}, 1 \mathrm{H}\right), 2.06$ $1.99(\mathrm{~m}, 1 \mathrm{H}), 1.89-1.76(\mathrm{~m}, 3 \mathrm{H}), 1.74-1.62(\mathrm{~m}, 4 \mathrm{H}), 1.61-1.51(\mathrm{~m}, 1 \mathrm{H})$, 1.42-1.36 (m, 1H), 1.32-1.26 (m, 1H), 1.21-1.09 (m, 1H), 0.93 (q, J = 5.1 $\mathrm{Hz}, 1 \mathrm{H})$.

${ }^{13} \mathrm{C}$ NMR $\left(100 \mathrm{MHz}, \mathrm{CDCl}_{3}\right) \delta=210.8,50.9,50.4,32.5,30.3,27.0,22.6$, $22.5,21.7,20.0$. 
HRMS (ESI): $\mathrm{m} / \mathrm{z}$ [M+Na] ${ }^{+}$calcd for $\mathrm{C}_{10} \mathrm{H}_{14} \mathrm{ONa}$ : 173.0937; found: 173.0939 .

\section{( \pm )-decahydrobenzo[ $a]$ cyclopropa[ $d][7]$ annulen-2(1H)-one $(3 c)$}

Scale: 0.19 g, 0.92 mmol; Yield: 0.130 g (86\%); Brown oil; $\mathrm{R}_{\mathrm{f}}=0.4$ (petroleum ether/EtOAc, 99:1).

IR (ATR): 2916, 2851, 1669, 1445, 1253, 1025, 848, $702 \mathrm{~cm}^{-1}$.

${ }^{1} \mathrm{H}$ NMR $\left(400 \mathrm{MHz}, \mathrm{CDCl}_{3}\right) \delta=2.08-1.99(\mathrm{~m}, 1 \mathrm{H}), 1.94-1.87(\mathrm{~m}, 3 \mathrm{H})$, $1.76-1.56(\mathrm{~m}, 6 \mathrm{H}), 1.54-1.48(\mathrm{~m}, 1 \mathrm{H}), 1.36-0.95(\mathrm{~m}, 7 \mathrm{H})$,

${ }^{13} \mathrm{C}$ NMR $\left(100 \mathrm{MHz}, \mathrm{CDCl}_{3}\right) \delta=212.8,50.4,38.1,35.1,33.4,30.3,27.6$, $26.1,25.1,25.0,19.7,12.5$.

HRMS (ESI): $\mathrm{m} / \mathrm{z}[\mathrm{M}+\mathrm{Na}]^{+}$calcd for $\mathrm{C}_{12} \mathrm{H}_{18} \mathrm{ONa}$ : 201.1250; found: 201.1251.

\section{Dinitrophenylhydrazone formation of 3a and 3c; General Procedure}

To a stirred solution of the ketone 3a-c ( 1 equiv.) in dry methanol $(0.08$ M) at rt under argon was added paratoluenosulfonic acid ( 0.05 equiv.). 2,4-dinitrophenylhydrazine (1.6 equiv.) was then added and the yellow solution was stirred at reflux for $3 \mathrm{~h}$, cooled down to rt and concentrated under reduced pressure. Purification by column chromatography on silica gel using petroleum ether/EtOAc (96/4) as eluent or recrystallization from ethanol afforded the desired product.

\section{( \pm )-(Z)-1-(2,4-dinitrophenyl)-2-((3R,5R)-tricyclo[5.2.0.0 3,5$]$ nonan- 2-ylidene)hydrazine (3a-DNPH)}

Scale: 0.056 g, 0.4 mmol; Yield: $0.088 \mathrm{~g}$ (68\%); Orange solid; $\mathrm{R}_{\mathrm{f}}=0.39$ (petroleum ether/EtOAc, 96:4); $\mathrm{mp}: 188^{\circ} \mathrm{C}$.

IR (ATR): 3284, 3102, 2920, 2850, 1613, 1585, 1519, 1499, 1416, 1331, $1305,1276,1221,1135,1071,920,832,741 \mathrm{~cm}^{-1}$.

$\left.{ }^{1} \mathrm{H} \mathrm{NMR} \mathrm{(400MHz,} \mathrm{CDCl}_{3}\right) \delta=9.14(\mathrm{~d}, J=2.6 \mathrm{~Hz}, 1 \mathrm{H}), 8.27$ (ddd, $J=9.6$, 2.6, $0.8 \mathrm{~Hz}, 1 \mathrm{H}), 7.92(\mathrm{~d}, J=9.6 \mathrm{~Hz}, 1 \mathrm{H}), 3.44-3.38(\mathrm{~m}, 1 \mathrm{H}, \mathrm{CHCN} \mathrm{cb}), 2.79-$ $2.69(\mathrm{~m}, 1 \mathrm{H}), 2.56-2.46(\mathrm{~m}, 1 \mathrm{H}), 2.43-2.36(\mathrm{~m}, 1 \mathrm{H}), 2.31-2.21(\mathrm{~m}, 2 \mathrm{H})$, 1.51-1.33 (m, 4H), 0.77-0.69 (m, 1H), $0.35(\mathrm{q}, J=4.4 \mathrm{~Hz}, 1 \mathrm{H})$.

${ }^{13} \mathrm{C}$ NMR $\left(100 \mathrm{MHz}, \mathrm{CDCl}_{3}\right) \delta=164.0,145.0,137.6,130.0,129.0,123.8$, 116.6, 38.5 (CHCN cb), 36.8, 30.8, 26.0, 24.2, 13.6, 13.5, 8.3 (CHCN cp).

HRMS (ESI): $\mathrm{m} / \mathrm{z}$ [M-H]+ calcd for $\mathrm{C}_{15} \mathrm{H}_{15} \mathrm{~N}_{4} \mathrm{O}_{4}:$ :315.1095; found: 315.1088.

\section{( \pm )-(Z)-1-(decahydrobenzo[a]cyclopropa[ $d][7]$ annulen-2(1H)-} ylidene)-2-(2,4-dinitrophenyl)hydrazine (3c-DNPH)

Scale: 0.083 g, $0.47 \mathrm{mmol}$; Yield: $0.120 \mathrm{~g}(71 \%)$; Orange red solid; $\mathrm{R}_{\mathrm{f}}=0.9$ (petroleum ether/EtOAc, 50:50); mp: $170{ }^{\circ} \mathrm{C}$.

IR (ATR): 3312, 2922, 2850, 1617, 1579, 1504, 1418, 1330, 1306, 1268, $1129,1070,920,836,743 \mathrm{~cm}^{-1}$.

$\left.{ }^{1} \mathrm{H} \mathrm{NMR} \mathrm{(400MHz}, \mathrm{CDCl}_{3}\right) \delta=11.67(\mathrm{~s}, 1 \mathrm{H}), 9.14(\mathrm{~d}, J=2.6 \mathrm{~Hz}, 1 \mathrm{H}), 8.27$ (dd, $J=9.6,2.6 \mathrm{~Hz}, 1 \mathrm{H}$ ), 7.93 (d, $J=9.6 \mathrm{~Hz}, 1 \mathrm{H}), 2.17-2.10(\mathrm{~m}, 1 \mathrm{H}), 2.01-$ $1.51(\mathrm{~m}, 10 \mathrm{H}), 1.36-1.23(\mathrm{~m}, 4 \mathrm{H}), 1.13-1.01(\mathrm{~m}, 2 \mathrm{H}), 0.73(\mathrm{q}, J=5.3 \mathrm{~Hz}$, $1 \mathrm{H})$.

${ }^{13} \mathrm{C}$ NMR $\left(100 \mathrm{MHz}, \mathrm{CDCl}_{3}\right) \delta=206.9,164.2,145.0,129.8,123.7,116.4$, $77.2,48.1,39.3,34.4,34.3,33.3,30.9,27.2,26.4,26.1,15.5,13.8,11.2$.

HRMS (ESI): $\mathrm{m} / \mathrm{z}$ [M-H] $]^{+}$calcd for $\mathrm{C}_{18} \mathrm{H}_{21} \mathrm{~N}_{4} \mathrm{O}_{4}$ : 357.1568; found: 357.1566.

\section{( \pm )-(2R,3R,5R)-2-(phenylethynyl)tricyclo[5.2.0.0 3,5$]$ nonan-2-ol (4)}

To a stirred solution of phenylacetylene $(0.06 \mathrm{~mL}, 0.5 \mathrm{mmol}, 1.1$ equiv.) in THF $(1.5 \mathrm{~mL})$ at $-78^{\circ} \mathrm{C}$ under argon was added $n \operatorname{BuLi}(0.18 \mathrm{~mL}, 2.5 \mathrm{M}$ in hexane, $0.46 \mathrm{mmol}, 1$ equiv.) dropwise and the mixture was stirred at - $78{ }^{\circ} \mathrm{C}$ for $1 \mathrm{~h}$. To this white suspension was cannulated ketone 3a ( $0.062 \mathrm{~g}, 0.46 \mathrm{mmol}, 1$ equiv.) in THF (1.5 mL) dropwise. The yellow suspension was allowed to warm to $\mathrm{rt}$ for $2 \mathrm{~h}$ and quenched with saturated ammonium chloride solution. The aqueous phase was extracted three times with diethyl ether. The combined organic layers were washed with brine, dried over $\mathrm{MgSO}_{4}$ and concentrated under reduced pressure. Purification by column chromatography on silica gel using pentane/ $\mathrm{Et}_{2} \mathrm{O}(97 / 3)$ as eluent afforded the desired product as only one diastereosomer.

Yield: 0.066 g (61\%); Colorless oil; $\mathrm{R}_{\mathrm{f}}=0.35$ (pentane/Et $20,95: 5$ ).

IR (ATR): 3564, 2925, 2853, 1489, 1442, 1148, 937, 753, $689 \mathrm{~cm}^{-1}$.

${ }^{1} \mathrm{H} \mathrm{NMR}\left(400 \mathrm{MHz}, \mathrm{CDCl}_{3}\right) \delta=7.45-7.41(\mathrm{~m}, 2 \mathrm{H}), 7.30-7.28(\mathrm{~m}, 3 \mathrm{H}), 2.79-$ $2.70(\mathrm{~m}, 1 \mathrm{H}, \mathrm{CHCO} \mathrm{cb}), 2.66-2.55(\mathrm{~m}, 1 \mathrm{H}), 2.24-2.07(\mathrm{~m}, 4 \mathrm{H}), 1.87(\mathrm{~s}, 1 \mathrm{H}$, $\mathrm{OH}), 1.64-1.53(\mathrm{~m}, 1 \mathrm{H}), 1.34-1.27(\mathrm{~m}, 1 \mathrm{H}, \mathrm{CHCO} \mathrm{cp}), 1.08-0.89(\mathrm{~m}, 2 \mathrm{H})$, 0.76-0.66 (m, 2H).

${ }^{13} \mathrm{C}$ NMR (100MHz, $\mathrm{CDCl}_{3}$ ) $\delta=131.8(2 \mathrm{C}), 128.3$ (2 C), 128.2, 123.1, 94.6, 82.5, 62.3, 42.9 (CHCO cb), 32.2, 27.2, 26.4, 20.4, 19.6 (СHCO cp), 10.0, 8.2 .

HRMS (ESI): $\mathrm{m} / \mathrm{z}$ [M+Na] ${ }^{+}$calcd for $\mathrm{C}_{17} \mathrm{H}_{18} \mathrm{ONa}$ : 261.1250; found: 261.1246.

( \pm )-(2R,3R,5R)-2-(allyloxy)-2-(phenylethynyl)tricyclo[5.2.0.0 3,5$]-$ nonane (4-0allyl)

To a stirred suspension of sodium hydride $(0.013 \mathrm{~g}, 60 \%$ in oil, 0.32 mmol, 1.5 equiv.), sodium iodide (0.003g, $0.021 \mathrm{mmol}, 0.1$ equiv.) in dry DMF ( $3.2 \mathrm{~mL}$ ) was added allyl bromide $(0.04 \mathrm{~mL}, 0.42 \mathrm{mmol}, 2$ equiv.) at $0{ }^{\circ} \mathrm{C}$ under argon. To this was cannulated alcohol $9(0.050 \mathrm{~g}, 0.21 \mathrm{mmol}$, 1 equiv.) in DMF $(0.5 \mathrm{~mL})$ and the reaction mixture was stirred at $\mathrm{rt}$ for $18 \mathrm{~h}$. The reaction was quenched with water $(15 \mathrm{~mL})$ and extracted three times with diethyl ether. The combined organic layers were washed with brine, dried over $\mathrm{MgSO}_{4}$ and concentrated under reduced pressure. Purification by column chromatography on silica gel using petroleum ether/EtOAc (98/2) as eluent afforded the desired product .

Yield: 0.046 g (79\%); Colorless oil; $\mathrm{R}_{\mathrm{f}}=0.29$ (petroleum ether/EtOAc, 98:2).

IR (ATR): 2927, 2853, 1734, 1645, 1490, 1443, 1256, 1142, 1060, 914, $753,689,618 \mathrm{~cm}^{-1}$.

${ }^{1} \mathrm{H} \mathrm{NMR}\left(400 \mathrm{MHz}, \mathrm{CDCl}_{3}\right) \delta=7.45-7.42(\mathrm{~m}, 2 \mathrm{H}), 7.31-7.29(\mathrm{~m}, 3 \mathrm{H}), 6.00$ (ddt, $J=17.2,10.3,5.1 \mathrm{~Hz}, 1 \mathrm{H}), 5.34(\mathrm{dq}, J=17.2,1.8 \mathrm{~Hz}, 1 \mathrm{H}), 5.14(\mathrm{dq}, J=$ $10.5,1.6 \mathrm{~Hz}, 1 \mathrm{H}$ ), 4.22 (qdt, $J=12.9,5.0,1.7 \mathrm{~Hz}, 2 \mathrm{H}), 2.92-2.86(\mathrm{~m}, 1 \mathrm{H}$, CHCO cb), 2.71-2.61 (m, 1H), 2.31-2.23 (m, 1H), 2.17-2.08 (m, 2H), 1.68$1.62(\mathrm{~m}, 1 \mathrm{H}), 1.27-1.19$ (m, 3H, CHCO cp), 1.05-0.99 (m, 1H), 0.78-0.73 $(\mathrm{m}, 2 \mathrm{H})$.

${ }^{13} \mathrm{C}$ NMR (100MHz, $\left.\mathrm{CDCl}_{3}\right) \delta=135.7,131.8$ (2 C), 128.3 (2 C), 128.1, 123.4, 115.5, 92.6, 84.8, 71.6, 65.5, 41.9 (CHCO cb), 31.4, 26.6, 25.7, 19.7, 18.5 (CHCO cp), 10.9, 9.2.

HRMS (ESI): $\mathrm{m} / \mathrm{z}$ [M+Na] ${ }^{+}$calcd for $\mathrm{C}_{20} \mathrm{H}_{22} \mathrm{ONa}$ : 301.1563; found: 301.1559.

\section{Halide cyclopropane opening; General Procedure}

To a stirred solution of tricycle ketone (1 equiv.) in acetonitrile ( $0.25 \mathrm{M})$ at $\mathrm{rt}$ under argon was added dry pyridinium hydrochloride (4 equiv.) portion wise and the reaction mixture was stirred at reflux for $12 \mathrm{~h}$. The reaction mixture was then poured into a saturated sodium chloride solution. The aqueous phase was extracted three times with 
diethylether. The combined organic layers were washed with brine, dried over $\mathrm{MgSO}_{4}$ and concentrated under reduced pressure. Purification by column chromatography on silica gel using pentane/Et ${ }_{2} \mathrm{O}(90 / 10)$ as eluent afforded the desired product.

\section{( \pm )-4-(chloromethyl)bicyclo[4.2.0]octan-2-one (5)}

Scale: 0.201 g, 1.48 mmol; Yield: 0.12 g (48\%); Yellow oil; $R_{f}=0.58$ (pentane/Et $20,60: 40$ ).

IR (ATR): 2942, 2863, 1697, 1442, 1290, 1199, 1056, 931, 723, 691, 563 $\mathrm{cm}^{-1}$.

${ }^{1} \mathrm{H} \mathrm{NMR}\left(400 \mathrm{MHz}, \mathrm{CDCl}_{3}\right) \delta=3.47\left(\mathrm{~d}, J=5.2 \mathrm{~Hz}, 2 \mathrm{H}, \mathrm{CH}_{2} \mathrm{Cl}\right), 3.03-2.96(\mathrm{~m}$, $1 \mathrm{H}, \mathrm{CHCO} \mathrm{cb}), 2.74-2.66(\mathrm{~m}, 1 \mathrm{H}), 2.51-2.47(\mathrm{~m}, 1 \mathrm{H}), 2.35-2.18(\mathrm{~m}, 4 \mathrm{H})$, 2.12-2.07 (m, 2H), 1.59-1.50 (m, 1H), 1.48-1.39 (m, 1H).

${ }^{13} \mathrm{C}$ NMR $\left(75 \mathrm{MHz}, \mathrm{CDCl}_{3}\right) \delta=213.1,49.3\left(\mathrm{CH}_{2} \mathrm{Cl}\right), 43.7$ (CHCO cb), 43.0 ( $\left.\mathrm{CH}_{2} \mathrm{CO}\right), 37.7,34.8,32.8,26.1,24.2$.

HRMS (ESI): m/z [M+Na] ${ }^{+}$calcd for $\mathrm{C}_{9} \mathrm{H}_{13} \mathrm{ClONa}$ : 195.0547; found: 195.0548.

\section{( \pm )-4-(bromomethyl)bicyclo[4.2.0]octan-2-one (6)}

Scale: 0.175 g, 1.28 mmol; Yield: 0.136 g (49\%); Brown oil; $R_{f}=0.8$ (petroleum ether/EtOAc, 50:50).

IR (ATR): 2935, 2856, 1698, 1445, 1275, 1222, 916, $638 \mathrm{~cm}^{-1}$.

$\left.{ }^{1} \mathrm{H} \mathrm{NMR} \mathrm{(400MHz,} \mathrm{CDCl}_{3}\right) \delta=3.37\left(\mathrm{~d}, J=5.5 \mathrm{~Hz}, 2 \mathrm{H}, \mathrm{CH}_{2} \mathrm{Br}\right), 3.04-2.97$ (m, 1H, CHCO cb), 2.77-2.67 (m, 1H), 2.56-2.50 (m, 1H), 2.38-2.06 (m, $6 \mathrm{H}), 1.62-1.53(\mathrm{~m}, 1 \mathrm{H}), 1.48-1.39(\mathrm{~m}, 1 \mathrm{H})$.

${ }^{13} \mathrm{C}$ NMR (75MHz, $\left.\mathrm{CDCl}_{3}\right) \delta=212.9,44.1(\mathrm{CHCO} \mathrm{cb}), 43.7\left(\mathrm{CH}_{2} \mathrm{CO}\right), 38.5$ $\left(\mathrm{CH}_{2} \mathrm{Br}\right), 37.5,34.8,34.0,26.2,24.2$.

HRMS (ESI): $\mathrm{m} / \mathrm{z}$ [M+Na] ${ }^{+}$calcd for $\mathrm{C}_{9} \mathrm{H}_{13} \mathrm{BrONa}$ : 239.0042; found: 239.0039 .

\section{( \pm )-5-(chloromethyl)-2-oxabicyclo[5-2.0]nonan-3-one (7)}

Scale: 0.100 g, 0.58 mmol; Yield: 0.096 g (84\%); Colorless oil; $\mathrm{R}_{\mathrm{f}}=0.22$ (cyclohexane/EtOAc, 80:20).

IR (ATR): 2948, 2859, 1730, 1454, 1359, 1273, 1186, 1148, 1069, 1017, $935,726 \mathrm{~cm}^{-1}$.

$\left.{ }^{1} \mathrm{H} \mathrm{NMR} \mathrm{(400MHz,} \mathrm{CDCl}_{3}\right) \delta=4.82(\mathrm{q}, J=7.2 \mathrm{~Hz}, 1 \mathrm{H}, \mathrm{CHO} \mathrm{cb}) 3.43-3.42$ (d, $J=7.3 \mathrm{~Hz}, 2 \mathrm{H}, \mathrm{CH}_{2} \mathrm{Cl}$ ), 2.74-2.67 (m, $\left.1 \mathrm{H}\right), 2.63-2.51\left(\mathrm{~m}, 2 \mathrm{H}, \mathrm{CH}_{2} \mathrm{CO}\right.$ ), 2.45-2.35 (m, 1H), 2.26-2.00 (m, 3H), 1.97-1.92 (m, 1H), 1.72-1.63 (m, 1H), $1.51-1.41(\mathrm{~m}, 1 \mathrm{H})$.

${ }^{13} \mathrm{C}$ NMR $\left(100 \mathrm{MHz}, \mathrm{CDCl}_{3}\right) \delta=171.2,75.1$ ( $\left.\mathrm{CHO} \mathrm{cb}\right), 47.0\left(\mathrm{CH}_{2} \mathrm{Cl}\right), 37.4$, 35.6, $35.0\left(\mathrm{CH}_{2} \mathrm{CO}\right), 31.4,25.8,21.2$.

HRMS (ESI): $\mathrm{m} / \mathrm{z}$ [M+Na] ${ }^{+}$calcd for $\mathrm{C}_{9} \mathrm{H}_{13} \mathrm{ClO}_{2} \mathrm{Na}$ : 211.0496; found: 211.0497.

\section{Regioselective Baeyer-Villiger oxidation; General Procedure}

To a stirred solution of tricycle ketone (1 equiv.) in dry dichloromethane $(0.17 \mathrm{M})$ at $0{ }^{\circ} \mathrm{C}$ under argon was added $m$ CPBA $(70 \%, 2.4$ equiv.) portion wise and the mixture was stirred at $\mathrm{rt}$ for $12 \mathrm{~h}$. The reaction mixture was quenched with $10 \% \mathrm{~K}_{2} \mathrm{CO}_{3}(4 \times 10 \mathrm{~mL})$ solution and saturated $\mathrm{Na}_{2} \mathrm{~S}_{2} \mathrm{O}_{3}(10 \mathrm{~mL})$ solution. The aqueous phase was extracted with dichloromethane. The combined organic layers were dried over $\mathrm{MgSO}_{4}$, filtered and concentrated under reduced pressure. Purification by column chromatography on silica gel using pentane/ $\mathrm{Et}_{2} \mathrm{O}(80 / 20)$ as eluent afforded the desired products.

$( \pm)-(3 R, 5 R)-7-o x a t r i c y c l o\left[6.2 .0 .0^{3,5}\right]$ decan-6-one (8)
Scale: 0.100 g, 0.73 mmol; Yield: $0.081 \mathrm{~g}$ (72\%); Yellow oil; $\mathrm{R}_{\mathrm{f}}=0.26$ (pentane/ $\mathrm{Et}_{2} \mathrm{O}, 80: 20$ ).

IR (ATR): 2941, 2860, 1724, 1288, 1174, 1115, 1032, $986 \mathrm{~cm}^{-1}$.

${ }^{1} \mathrm{H}$ NMR $\left(400 \mathrm{MHz}, \mathrm{CDCl}_{3}\right) \delta=4.97-4.93(\mathrm{~m}, 1 \mathrm{H}, \mathrm{CHO} \mathrm{cb}), 2.79-2.69(\mathrm{~m}$, $1 \mathrm{H}), 2.33-2.25(\mathrm{~m}, 1 \mathrm{H}), 2.23-2.17(\mathrm{~m}, 1 \mathrm{H}), 2.00-1.89(\mathrm{~m}, 3 \mathrm{H}), 1.77-1.71$ (m, 1H, CHCO cp), 1.03-0.93 (m, 2H), 0.78-0.68 (m, 1H), 0.62-0.56 (m, $1 \mathrm{H})$.

${ }^{13} \mathrm{C}$ NMR $\left(100 \mathrm{MHz}, \mathrm{CDCl}_{3}\right) \delta=173.4,78.1$ (CHO cb), 39.7, 31.8, 25.4, 25.2, 17.9 (CHCO cp), 12.6, 11.7.

HRMS (ESI): m/z [M+Na] ${ }^{+}$calcd for $\mathrm{C}_{9} \mathrm{H}_{12} \mathrm{O}_{2} \mathrm{Na}$ : 175.0729; found: 175.0730 .

$( \pm)-(1 \mathrm{aR}, 7 \mathrm{aR})-$ octahydrocyclopenta[b]cyclopropa[e]oxepin-2(1H)one (9)

Scale: 0.100 g, $0.67 \mathrm{mmol}$; Yield: $0.024 \mathrm{~g}$ (22\%); Yellow oil; $\mathrm{R}_{\mathrm{f}}=0.2$ (pentane/Et ${ }_{2} \mathrm{O}, 85: 15$ ).

IR (ATR): 2945, 2867, 1693, 1451, 1275, 1207, 1072, 1052, $866 \mathrm{~cm}^{-1}$.

${ }^{1} \mathrm{H} \mathrm{NMR}\left(400 \mathrm{MHz}, \mathrm{CDCl}_{3}\right) \delta=4.32-4.27(\mathrm{~m}, 1 \mathrm{H}, \mathrm{CHO} \mathrm{cpe}), 2.43$ (ddd, $J=$ $13.9,9.1,4.6 \mathrm{~Hz}, 1 \mathrm{H}), 2.12-1.91(\mathrm{~m}, 4 \mathrm{H}), 1.85-1.78(\mathrm{~m}, 2 \mathrm{H}), 1.74-1.64(\mathrm{~m}$, 1H), $1.60-1.47(\mathrm{~m}, 2 \mathrm{H}), 1.29-1.13(\mathrm{~m}, 3 \mathrm{H})$.

${ }^{13} \mathrm{C}$ NMR (100MHz, $\left.\mathrm{CDCl}_{3}\right) \delta=174.3,82.2$ (CHO cpe), 45.3, 34.2, 32.7, 31.0, 22.4, 22.1 (CHCO cp), 18.4, 12.5 .

HRMS (ESI): m/z [M+Na] calcd for $\mathrm{C}_{10} \mathrm{H}_{14} \mathrm{O}_{2} \mathrm{Na}$ : 189.0886; found: 189.0889 .

\section{Oxime formation; General Procedure}

The tricyclic ketone (1 equiv.) and hydroxylammonium hydrochloride (1.7 equiv.) were dissolved in ethanol $(0.98 \mathrm{M})$ and heated to $60^{\circ} \mathrm{C}$. After addition of a solution of $\mathrm{Na}_{2} \mathrm{CO}_{3}$ (0.7 equiv.) in water (4.9 M), the reaction was stirred at $60^{\circ} \mathrm{C}$ until TLC indicated complete conversion. After cooling to $\mathrm{rt}$, the mixture was quenched with water and extracted with EtOAc. The combined organic layers were washed with brine, dried over $\mathrm{MgSO}_{4}$ and concentrated under reduced pressure. Purification by column chromatography on silica gel using petroleum ether/EtOAc $(6 / 1)$ as eluent afforded the desired product.

\section{( \pm )-(3R,5R)-tricyclo[5.2.0.0 3,5$]$ nonan-2-one oxime (10)}

Scale: $0.200 \mathrm{~g}, 1.47 \mathrm{mmol}$; Yield: $0.113 \mathrm{~g}(51 \%)$ of a mixture of two diastereomers Z/E (1:1); Yellow oil; $\mathrm{R}_{\mathrm{f}}=0.1$ (petroleum ether/EtOAc, 6:1).

IR (ATR): 3214, 2927, 2851, 1634, 1447, 1356, 1089, 984, 908, $727 \mathrm{~cm}^{-1}$.

${ }^{1} \mathrm{H} \mathrm{NMR}\left(600 \mathrm{MHz}, \mathrm{CDCl}_{3}\right) \delta=3.57(\mathrm{td}, J=9.8,6.9 \mathrm{~Hz}, 1 \mathrm{H}), 3.19(\mathrm{td}, J=$ 10.0, $6.7 \mathrm{~Hz}, 1 \mathrm{H}), 2.76-2.70(\mathrm{~m}, 1 \mathrm{H}), 2.69-2.63(\mathrm{~m}, 1 \mathrm{H}), 2.54-2.48(\mathrm{~m}, 1 \mathrm{H})$, 2.39-2.33 (m, 1H), 2.20-2.04 (m, 5H), 2.01-1.96 (m, 1H), 1.94-1.88 (m, $1 \mathrm{H}), 1.70-1.62(\mathrm{~m}, 3 \mathrm{H}), 1.43-1.38(\mathrm{~m}, 3 \mathrm{H}), 1.36-1.32(\mathrm{~m}, 1 \mathrm{H}), 1.25(\mathrm{~s}, 1 \mathrm{H}$, $\mathrm{OH}), 1.16-1.13(\mathrm{~m}, 1 \mathrm{H}), 1.07(\mathrm{q}, J=5.1 \mathrm{~Hz}, 1 \mathrm{H}), 0.98(\mathrm{td}, J=8.8,5.1 \mathrm{~Hz}$, $1 \mathrm{H}), 0.87(\mathrm{q}, J=5.1 \mathrm{~Hz}, 1 \mathrm{H})$.

${ }^{13} \mathrm{C}$ NMR $\left(151 \mathrm{MHz}, \mathrm{CDCl}_{3}\right) \delta=162.7,162.4,34.2,32.5,31.5,30.8,26.7$, $26.1,25.3,24.8,24.6,24.6,15.0,13.5,12.9,12.8,12.7,9.1$.

HRMS (ESI): $\mathrm{m} / \mathrm{z}[\mathrm{M}+\mathrm{H}]^{+}$calcd for $\mathrm{C}_{9} \mathrm{H}_{14} \mathrm{NO}: 152.1070$; found: 152.1073 .

( \pm )-decahydrobenzo[a]cyclopropa[d][7]annulen-2(1H)-one oxime (13)

Scale: $0.130 \mathrm{~g}, 0.73 \mathrm{mmol}$; Yield: $0.031 \mathrm{~g}(22 \%)$ of a mixture of two diastereomers Z/E (1:1); $0.051 \mathrm{~g}(36 \%)$ of a single diastereomer; 
combined yield: $58 \%$; Colorless solids; $\mathrm{R}_{\mathrm{f}}=0.63,0.56$ (petroleum ether/EtOAc, 50:50).

Analysis of one diastereomer:

Mp: $120-125^{\circ} \mathrm{C}$

IR (ATR): 3286, 2921, 2849, 1636, 1446, 1313, 1257, 1029, 943, 780, $656 \mathrm{~cm}^{-1}$.

${ }^{1} \mathrm{H} \mathrm{NMR}\left(300 \mathrm{MHz}, \mathrm{CDCl}_{3}\right) \delta=8.20(\mathrm{br} \mathrm{s}, 1 \mathrm{H}), 2.65(\mathrm{tdd}, J=11.5,3.6,1.1$ $\mathrm{Hz}, 1 \mathrm{H}), 2.10-2.03(\mathrm{~m}, 1 \mathrm{H}), 1.99-1.89(\mathrm{~m}, 2 \mathrm{H}), 1.77-1.56(\mathrm{~m}, 5 \mathrm{H}), 1.38-$ $1.24(\mathrm{~m}, 4 \mathrm{H}), 0.96-0.80(\mathrm{~m}, 3 \mathrm{H}), 0.76-0.63(\mathrm{~m}, 1 \mathrm{H}), 0.53(\mathrm{q}, J=4.8 \mathrm{~Hz}$, $1 \mathrm{H})$.

${ }^{13} \mathrm{C} \mathrm{NMR}\left(75 \mathrm{MHz}, \mathrm{CDCl}_{3}\right) \delta=164.5,42.0,37.3,34.0,29.5,26.6,26.4,26.1$, $15.8,12.0,10.7$.

HRMS (ESI): $\mathrm{m} / \mathrm{z}[\mathrm{M}+\mathrm{Na}]^{+}$calcd for $\mathrm{C}_{12} \mathrm{H}_{19} \mathrm{NONa}$ : 216.1359; found: 216.1354 .

\section{Beckmann Rearrangment; General Procedure}

To a solution of the oxime (1 equiv.) in 1,4-dioxane ( $0.15 \mathrm{M})$ were added water $(0.11 \mathrm{M}), \mathrm{NaOH}$ (5 equiv.) and $p$ - $\mathrm{TsCl}$ (2 equiv.). The reaction was stirred at rt for $19 \mathrm{~h}$, quenched with water and extracted with DCM. The combined organic layers were washed with brine, dried over $\mathrm{MgSO}_{4}$ and concentrated under reduced pressure. Purification by column chromatography on silica gel using petroleum ether/EtOAc (50/50 to pure EtOAc) afforded the desired products in two separated fractions.

\section{$( \pm)-(1 S, 3 R, 5 R, 8 R)-6$-azatricyclo[6.2.0.0 3,5$]$ decan-7-one (11)}

Scale: $0.113 \mathrm{~g}$ of 11, $0.75 \mathrm{mmol}$; Yield: $0.023 \mathrm{~g}(20 \%)$; Colorless solid; $\mathrm{R}_{\mathrm{f}}$ $=0.3$ (petroleum ether $/$ EtOAc, $50: 50$ ); $\mathrm{mp} 136^{\circ} \mathrm{C}$.

IR (ATR): 2922, 2853, 1645, 1399, 1344, 1279, 1211, 1032, 875, 801, $675 \mathrm{~cm}^{-1}$.

$\left.{ }^{1} \mathrm{H} \mathrm{NMR} \mathrm{(400MHz,} \mathrm{CDCl}_{3}\right) \delta=5.87$ (br s, $\left.1 \mathrm{H}\right), 3.23-3.20(\mathrm{~m}, 1 \mathrm{H}), 2.86-$ $2.80(\mathrm{~m}, 1 \mathrm{H}), 2.48-2.45(\mathrm{~m}, 1 \mathrm{H}), 2.39-2.36(\mathrm{~m}, 1 \mathrm{H}), 2.15-2.13(\mathrm{~m}, 1 \mathrm{H})$, 2.06-2.00 (m, 1H), 1.97-1.92 (m, 1H), 1.63 (p, J= 10.1 Hz, 1H), 0.98-0.88 $(\mathrm{m}, 3 \mathrm{H}), 0.36(\mathrm{q}, J=4.6 \mathrm{~Hz}, 1 \mathrm{H})$.

${ }^{13} \mathrm{C}$ NMR $\left(151 \mathrm{MHz}, \mathrm{CDCl}_{3}\right) \delta=175.7,41.5,36.0,32.4,26.7,25.0,19.6$, $16.7,16.1$.

HRMS (ESI): $\mathrm{m} / \mathrm{z}[\mathrm{M}+\mathrm{Na}]^{+}$calcd for $\mathrm{C}_{9} \mathrm{H}_{13} \mathrm{NONa}$ : 174.0889; found: 174.0891.

\section{$( \pm)-(1 S, 3 R, 5 R, 8 R)-7-a z a t r i c y c l o\left[6.2 .0 .0^{3,5}\right]$ decan-6-one (12)}

Scale: $0.113 \mathrm{~g}$ of 11, $0.75 \mathrm{mmol}$; Yield: $0.026 \mathrm{~g}$ (23\%); Colorless solid; $\mathrm{R}_{\mathrm{f}}$ $=0.1$ (petroleum ether $/$ EtOAc, 50:50); $\mathrm{mp} .125-132^{\circ} \mathrm{C}$.

IR (ATR): 3273, 3194, 3063, 2994, 2928, 2854, 1643, 1406, 1338, 1303, $1239,1158,1022,921,789,698,649 \mathrm{~cm}^{-1}$.

$\left.{ }^{1} \mathrm{H} \mathrm{NMR} \mathrm{(400MHz,} \mathrm{CDCl}_{3}\right) \delta=5.45$ (br s, $\left.1 \mathrm{H}\right), 4.17-4.14(\mathrm{~m}, 1 \mathrm{H}), 2.68-$ $2.58(\mathrm{~m}, 1 \mathrm{H}), 2.43-2.34(\mathrm{~m}, 1 \mathrm{H}), 2.23-2.17(\mathrm{~m}, 1 \mathrm{H}), 2.09-2.01(\mathrm{~m}, 1 \mathrm{H})$, $1.89-1.79(\mathrm{~m}, 1 \mathrm{H}), 1.63-1.53(\mathrm{~m}, 2 \mathrm{H}), 0.97-0.78(\mathrm{~m}, 3 \mathrm{H}), 0.54(\mathrm{q}, J=4.6$ $\mathrm{Hz}, 1 \mathrm{H})$.

${ }^{13} \mathrm{C} \mathrm{NMR}\left(151 \mathrm{MHz}, \mathrm{CDCl}_{3}\right) \delta=174.3,50.9,37.7,32.9,26.0,25.4,18.2$, $12.5,11.1$.

HRMS (ESI): $\mathrm{m} / \mathrm{z}[\mathrm{M}+\mathrm{Na}]^{+}$calcd for $\mathrm{C}_{9} \mathrm{H}_{13} \mathrm{NONa}$ : 174.0889; found: 174.0892 .

( \pm )- $(1 \mathrm{aR}, 3 \mathrm{aS}, 7 \mathrm{aS}, 9 \mathrm{aR})$-dodecahydro-3H-benzo[f]cyclopropa[b]azocin-3-one (14)
Scale: $0.034 \mathrm{~g}$ of 15, $0.18 \mathrm{mmol}$, using 6.3 equiv. of $\mathrm{NaOH}$; Yield: $0.008 \mathrm{~g}$ (22\%); Colorless solid; $\mathrm{R}_{\mathrm{f}}=0.21$ (petroleum ether/EtOAc, 50:50); mp. $132-134^{\circ} \mathrm{C}$

IR (ATR): 3262, 3192, 3054, 2996, 2917, 2846, 1633, 1446, 1407, 1344, 1292, 1234, 1172, 1112, 1079, 1033, 964, 880, 829, 785, 746, 705, 648 $\mathrm{cm}^{-1}$.

${ }^{1} \mathrm{H} \mathrm{NMR}\left(400 \mathrm{MHz}, \mathrm{CDCl}_{3}\right) \delta=5.45(\mathrm{br} \mathrm{s}, 1 \mathrm{H}), 2.70(\mathrm{td}, J=6.8,3.2 \mathrm{~Hz}, 1 \mathrm{H})$, 2.34-2.21 (m, 1H), 2.08-1.97 (m, 3H), 1.83-1.72 (m, 2H), 1.65-1.43 (m, $4 \mathrm{H}), 1.41-1.31(\mathrm{~m}, 2 \mathrm{H}), 1.18-1.05(\mathrm{~m}, 1 \mathrm{H}), 1.03-0.96(\mathrm{~m}, 2 \mathrm{H}), 0.94-0.84$ (m, 1H), 0.24 (ddd, $J=6.5,5.3,3.3 \mathrm{~Hz}, 1 \mathrm{H}$ ).

${ }^{13} \mathrm{C}$ NMR $\left(75 \mathrm{MHz}, \mathrm{CDCl}_{3}\right) \delta=181.9,54.3,36.9,35.4,35.3,33.7,27.3,26.7$, 26.6, 23.4, 20.6, 15.2 .

HRMS (ESI): $\mathrm{m} / \mathrm{z}$ [M+Na $]^{+}$calcd for $\mathrm{C}_{12} \mathrm{H}_{19} \mathrm{NONa:} 216.1359$; found: 216.1362.

\section{( \pm )-(1aR,3aS,7aS,9aR)-dodecahydro-2H-benzo[b]cyclopropa[f]- azocin-2-one (15)}

Scale: $0.034 \mathrm{~g}$ of 13, $0.18 \mathrm{mmol}$, using 6.3 equiv. of $\mathrm{NaOH}$; Yield: $0.005 \mathrm{~g}$ (14\%); Colorless solid; $\mathrm{R}_{\mathrm{f}}=0.16$ (petroleum ether/EtOAc, 50:50); mp. $114-118{ }^{\circ} \mathrm{C}$

IR (ATR): 3273, 3192, 3054, 3000, 2921, 2850, 1639, 1492, 1446, 1424, $1412,1332,1269,1085,1079,1027,1085,1079,1027,935,857,813$, $800,729,695,636 \mathrm{~cm}^{-1}$.

${ }^{1} \mathrm{H} \mathrm{NMR}\left(300 \mathrm{MHz}, \mathrm{CDCl}_{3}\right) \delta=5.88(\mathrm{br} \mathrm{s}, 1 \mathrm{H}), 5.77(\mathrm{br} \mathrm{s}, 1 \mathrm{H}), 4.19(\mathrm{~d}, J=$ $9.9 \mathrm{~Hz}, 1 \mathrm{H}), 2.70-2.59(\mathrm{~m}, 1 \mathrm{H}), 2.48-2.37(\mathrm{~m}, 1 \mathrm{H}), 2.17-2.04(\mathrm{~m}, 2 \mathrm{H})$, 1.92-1.69 (m, 13H), 1.56-1.43 (m, 3H), 1.33-1.23 (m, 5H), 1.05-0.85 (m, $8 \mathrm{H}), 0.74(\mathrm{~d}, J=5.0 \mathrm{~Hz}, 1 \mathrm{H}), 0.55(\mathrm{q}, J=5.9 \mathrm{~Hz}, 1 \mathrm{H})$.

${ }^{13} \mathrm{C}$ NMR $\left(100 \mathrm{MHz}, \mathrm{CDCl}_{3}\right) \delta=173.8,173.2,61.6,49.1,43.1,38.5,37.7$, 35.6, 34.6, 31.3, 30.1, 27.0, 26.9, 26.0, 25.9, 25.7, 25.1, 21.1, 20.1, 20.0, $18.4,16.7,10.9,7.7$.

HRMS (ESI): $\mathrm{m} / \mathrm{z}[\mathrm{M}+\mathrm{Na}]^{+}$calcd for $\mathrm{C}_{12} \mathrm{H}_{19} \mathrm{NONa:} 216.1359$; found: 216.1355 .

\section{Acknowledgment}

The authors thank UPMC, CNRS and IUF. This work was supported by LabEx MiChem (Ph. D. grant for SG) part of French state funds managed by the ANR within the Investissements d'Avenir programme under reference ANR-11-IDEX-0004-02. A postdoctoral research grant to MB from the department of science and technology of the French embassy in Germany is gratefully acknowledged. We also thank Lise-Marie Chamoreau and Geoffrey Gontard for the XRD analyses (IPCM) and Ange Dole for running some experiments.

\section{Supporting Information}

${ }^{1} \mathrm{H} \&{ }^{13} \mathrm{C}$ NMR spectra and crystal data.

\section{References}

(1) For pioneering discoveries, see: (a) Strickler, H.; Davis, J. B.; Ohloff, G. Helv. Chim. Acta 1976, 59, 1328. (b) Rautenstrauch, V. J. Org. Chem. 1984, 49, 950.

(2) Mainetti, E.; Mouriès, V.; Fensterbank, L.; Malacria, M.; MarcoContelles, J. Angew. Chem. Int. Ed. 2002, 41, 2132.

(3) (a) Fürstner, A. Chem. Soc. Rev. 2009, 38, 3208; (b) Fensterbank, L.; Malacria, M. Acc. Chem. Res. 2014, 47, 953. (c) Dorel, R.; Echavarren, A. M. Chem. Rev. 2015, 115, 9028.

(4) For recent reviews on propargyl acetate migrations, see: (a) Fensterbank, L.; Goddard, J.-P.; Malacria M.; Simonneau A. In Gold Catalysis: An Homogenous Approach: Toste, F. D.; Michelet, V., Eds.; Imperial College Press, London, 2014, p. 331. (b) Shiroodi, R. K.; 
Gevorgyan, V. Chem. Soc. Rev. 2013, 42, 4991. (c) Day, P. D.; Chan, P. W. H. Adv. Synth. Catal. 2016, 358, 1368.

(5) For seminal reports, see: (a) Mamane, V.; Gress, T.; Krause, H.; Fürstner, A. J. Am. Chem. Soc. 2004, 126, 8654. (b) Johansson, M. J.; Gorin, D. J.; Staben, S. T.; Toste, F. D. J. Am. Chem. Soc. 2005, 127, 18002. (c) Marion, N.; de Frémont, P.; Lemière, G.; Stevens, E. D.; Fensterbank, L.; Malacria, M.; Nolan, S. P. Chem. Commun. 2006, 2048.

(6) (a) Shu, X.-z.; Schienebeck, C. M.; Song, W.; Guzei, I. A.; Tang, W. Angew. Chem. Int. Ed. 2013, 52, (b) Fukuyama, T.; Ohta, Y.; Brancour, B.; Miyagawa, K.; Ryu, I.; Dhimane, A.-L.; Fensterbank, L.; Malacria, M. Chem. Eur. J. 2012 18, 7243; Shu, X.-Z.; Shu, D.; Schienebeck, C. M.; Tang, W. Chem. Soc. Rev. 2012, 41, 7698 and references therein.

(7) (a) Amjun, S.; Marco-Contelles, J. Tetrahedron 2005, 61, 4793. (b) Fürstner, A.; Hannen, P. Chem. Eur. J. 2006, 12, 3006. (c) C. Fehr, B. Winter, I. Magpantay, Chem. Eur. J. 2009, 15, 9773. (d) C. Fehr, M. Vuagnoux, A. Buzas, J. Arpagaus, J.; Sommer, H. Chem. Eur. J. 2011, 17, 6214. (e) Watson, I. D. G.; Ritter, S.; Toste, F. D. J. Am. Chem. Soc. 2009, 131, 2056. (f) Rao, W.; Chan, P. W. H. Chem. Eur. J. 2014, 20, 713.

(8) (a) Cariou, K.; Mainetti, E.; Fensterbank, L.; Malacria, M. Tetrahedron 2004, 60, 9745. (b) Harrak, Y.; Blaszykowski, C.; Bernard, M.; Cariou, K.; Mainetti, E.; Mouriès, V.; Dhimane, A.-L.; Fensterbank, L.; Malacria, M. J. Am. Chem. Soc. 2004, 126, 8656. (c) Blaszykowski, C.; Harrak, H.; Gonçalves, M.-H.; Cloarec, J.-M.; Dhimane, A.-L., Fensterbank, L.; Malacria, M. Org. Lett. 2004, 6, 3771. (d) Moreau, X.; Goddard, J.-P.; Bernard, M.; Lemière, G.; López-Romero, J. M.; Mainetti, E.; Marion, N.; Mouriès, V.; Thorimbert, S.; Fensterbank, L.; Malacria, M. Adv. Synth. Catal. 2008, 350, 43 .

(e) Moreau, X.; Hours, A.; Fensterbank, L.; Goddard, J.-P.; Malacria, M.; Thorimbert, S. J. Organomet. Chem. 2009, 694, 561-565. (f) Harrak, Y.; Makhlouf, M.; Azzaro, S.; Mainetti, E.; Lopez Romero, J. M.; Cariou, K.; Gandon, V.; Goddard, J.-P.; Malacria, M.; Fensterbank, L. J. Organometallic Chem. 2011, 696, 388.

(9) For mechanistic insights, see: (a) Soriano, E.; Marco-Contelles, J. Chem. Eur. J. 2008, 14, 6771. (b) Marion, N.; Lemière, G.; Correa, A.; Ramon, R. S.; Moreau, X.; de Frémont, P.; Dahmane, R.; Hours, A.; Lesage, D.; Tabet, J.-C.; Goddard, J.-P.; Gandon, V.; Cavallo, L.; Fensterbank, L.; Malacria, M.; Nolan, S. P. Chem. Eur. J. 2009, 15, 3243 and refs 7b-d.

(10) Mino, T.; Masuda, S.; Nishio, M.; Yamashita, M. J. Org. Chem. 1997, 62, 2633.

(11) 3a-DNPH CCDC 1481737; 3c-DNPH CCDC 1481738.

(12) Gordon, H. L.; Freeman, S.; Hudlicky, T. Synlett 2005, 2911.

(13) (a) Di Beello, N.; Pellacani, L.; Tardella, P. A. Synthesis 1978, 227. See also: (b) Huang, H.; Forsyth, C. J. Tetrahedron 1997, 53, 48, 16341.

(14) Demuth, M.; Raghavan, Helv. Chim. Acta 1979, 62, 2338.

(15) Wiberg, K. B.; Snoonian, J. R. J. Org. Chem. 1998, 63, 1390.

(16) In forcing conditions (5 equiv. of $m \mathrm{CPBA}, 3$ days reaction time), $50 \%$ conversion was observed giving presumable $\mathrm{BV}$ products, but not very cleanly.

(17) (a) Beckmann, E. Berichte der deutschen chemischen Gesellschaft 1886, 19, 988. (b) Winter, D. K.; Drouin, A.; Lessard, J.; Spino, C. J. Org. Chem. 2010, 75, 2610. (c) Donaruma, L. G.; Heldt, W. Z. Org. React. 2011, 11:1, 1-156. (d) Tandon, V. K.; Awasthi, A. K.; Maurya, H. K.; Mishra, P. J. Heterocycl. Chem. 2012, 49, 424. (e) Zhang, J.; Dong, C.; Du, C.; Luo, G. Org. Proc. Res.\& Dev. 2015, 19, 352.

(18) Kotha, S.; Ravikumar, O.; Majhi, J. Beilstein J. Org. Chem. 2015, 11, 1503.

(19) 13 CCDC 1481739

(20) Olah, G. A.; Fung A. P. Org. Synth. 1985, 63, 188. 